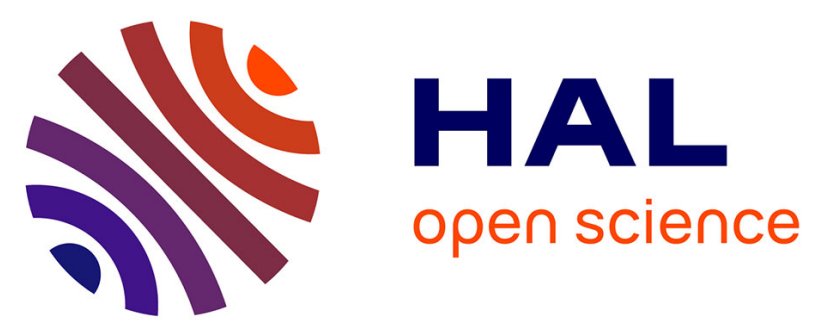

\title{
A contactless gesture interaction system using LTE (4G) signals
}

Weiyan Chen, Kai Niu, Dan Wu, Deng Zhao, Leye Wang, Daqing Zhang

\section{To cite this version:}

Weiyan Chen, Kai Niu, Dan Wu, Deng Zhao, Leye Wang, et al.. A contactless gesture interaction system using LTE (4G) signals. UbiComp/ISWC 2019 Adjunct: 2019 ACM international joint conference on Pervasive and Ubiquitous Computing and 2019 ACM International Symposium on Wearable Computers, Sep 2019, London, United Kingdom. pp.260-263, 10.1145/3341162.3343824 . hal-03002748

\section{HAL Id: hal-03002748 https://hal.science/hal-03002748}

Submitted on 12 Nov 2020

HAL is a multi-disciplinary open access archive for the deposit and dissemination of scientific research documents, whether they are published or not. The documents may come from teaching and research institutions in France or abroad, or from public or private research centers.
L'archive ouverte pluridisciplinaire HAL, est destinée au dépôt et à la diffusion de documents scientifiques de niveau recherche, publiés ou non, émanant des établissements d'enseignement et de recherche français ou étrangers, des laboratoires publics ou privés. 
See discussions, stats, and author profiles for this publication at: https://www.researchgate.net/publication/335542429

\section{Demo: A Contactless Gesture Interaction System Using LTE (4G) Signals}

Conference Paper · September 2019

DOI: $10.1145 / 3341162.3343824$

CITATION

1

6 authors, including:

\section{Weiyan Chen}

Peking University

3 PUBLICATIONS 5 CITATIONS

SEE PROFILE

D. Daqing Zhang

Institut Mines-Télécom

326 PUBLICATIONS 12,515 CITATIONS

SEE PROFILE

Some of the authors of this publication are also working on these related projects:

Project Contactless Human Sensing with WiFi CSI View project

Project Wi-Fi Sensing Project View project
READS

209

Niu Kai

Peking University

12 PUBLICATIONS 116 CITATIONS

SEE PROFILE 


\section{Demo: A Contactless Gesture Interaction System Using LTE (4G) Signals}

\author{
Weiyan Chen \\ Peking University \\ Peking University Information \\ Technology Institute (Tianjin Binhai) \\ Beijing, China \\ chenwy1117@pku.edu.cn \\ Deng Zhao \\ China University of Geosciences \\ (Beijing) \\ Beijing, China \\ dengzhao.cugb@gmail.com
}

\author{
Kai Niu \\ Peking University \\ Peking University Information \\ Technology Institute (Tianjin Binhai) \\ Beijing, China \\ xjtunk@pku.edu.cn \\ Leye Wang \\ Peking University \\ Beijing, China \\ leyewang@pku.edu.cn
}

\author{
Dan Wu \\ Peking University \\ Peking University Information \\ Technology Institute (Tianjin Binhai) \\ Beijing, China \\ dan@pku.edu.cn \\ Daqing Zhang \\ Peking University, Beijing \\ Telecom SudParis, Evry \\ China/France \\ dqzhang@sei.pku.edu.cn
}

\begin{abstract}
Nowadays, $4 \mathrm{G}$ devices are pervasive and most of the homes and offices in modern cities are covered by LTE signals. While it is very attractive to leverage ubiquitous LTE signals and use hand gestures to control the home appliances remotely, there is no work on such contactless gesture interaction systems reported yet. In this work, we present an LTE-based contactless gesture interaction system to recognize various hand gestures around a $4 \mathrm{G}$ terminal like mobile phone, which can be used to control the switch, channel and volume of a TV set remotely without holding any devices. The results show that the proposed system can recognize different hand gestures accurately leveraging LTE signals without training, and achieve remote TV control in real time in different settings.
\end{abstract}

\section{CCS CONCEPTS}

- Human-centered computing $\rightarrow$ Gestural input; Ubiquitous and mobile computing systems and tools.

\section{KEYWORDS}

LTE Signal; 4G Signal; Channel State Information (CSI); Gesture Interaction; Contactless Sensing

\footnotetext{
Permission to make digital or hard copies of part or all of this work for personal or classroom use is granted without fee provided that copies are not made or distributed for profit or commercial advantage and that copies bear this notice and the full citation on the first page. Copyrights for thirdparty components of this work must be honored. For all other uses, contact the owner/author(s).

UbiComp/ISWC '19 Adjunct, September 9-13, 2019, London, United Kingdom (c) 2019 Copyright held by the owner/author(s).

ACM ISBN 978-1-4503-6869-8/19/09.

https://doi.org/10.1145/3341162.3343824
}

ACM Reference Format:

Weiyan Chen, Kai Niu, Dan Wu, Deng Zhao, Leye Wang, and Daqing Zhang. 2019. Demo: A Contactless Gesture Interaction System Using LTE (4G) Signals. In Adjunct Proceedings of the 2019 ACM International foint Conference on Pervasive and Ubiquitous Computing and the 2019 International Symposium on Wearable Computers (UbiComp/ISWC '19 Adjunct), September 9-13, 2019, London, United Kingdom. ACM, New York, NY, USA, 4 pages. https: //doi.org/10.1145/3341162.3343824

\section{INTRODUCTION}

With the rapid development of LTE (4G) technology in recent years, $4 \mathrm{G}$ terminals like mobile phones have been widely used for communication and LTE $(4 \mathrm{G})$ signals have covered both indoor and outdoor environments in modern cities. Inspired by the advances in wireless sensing that have enabled a large variety of new applications such as indoor localization [4], trajectory tracking [5] and gesture recognition [3, 16], this work aims to equip the popular $4 \mathrm{G}$ terminals with gesture recognition capabilities, enabling contactless and remote control of home appliances. Unlike gesture recognition work using cameras [6], acoustic [3, 10] and WiFi [7] signals in mobile devices, LTE-based gesture recognition system expects to work well even in poor lighting conditions and wider sensing range distance from the transceivers for remote control. Different from the existing work on LTEbased human sensing using machine learning approaches for positioning $[2,8]$ and crowd density estimation [1], this work leverages the LTE signal propagation model to achieve robust and accurate gesture recognition without training.

In this work, we propose an LTE-based contactless gesture interaction system. Based on the Fresnel Zone model $[9,11-$ 15] that quantifies the relationship between the received signal variations and target movements, we design several 


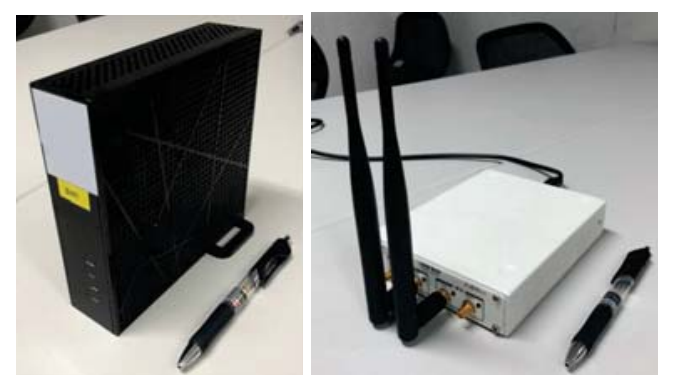

(a)

(b)

Figure 1: The transmitter and receiver of the system. (a) LTE base station: an indoor femtocell. (b) 4G terminal: a USRP B210 platform.

hand gestures that produce unique and repeatable signal variation patterns in the $4 \mathrm{G}$ terminal by carefully controlling the relative position of hand gestures to the LTE transceiver pair. In this way, the system could recognize these hand gestures accurately without the need of repeated offline-training in different deployments. Then, the recognized hand gestures are mapped to proper commands which are used to control the home appliances such as a TV set. We conduct extensive experiments with four participants in different environments. The results show that the proposed LTE-based gesture interaction system can recognize hand gestures reliably and achieve remote TV control in real time. Note that, $5 \mathrm{G}$ devices follow the same work scheme as $4 \mathrm{G}$ devices, so the proposed LTE-based gesture intraction system can be easily migrated to $5 \mathrm{G}$ devices with little efforts.

\section{DEMONSTRATION SYSTEM}

In this section, we first introduce the system setup. Then we present the LTE signal propagation model which describing how the signal propagates from a transmitter to a receiver and how it is modulated by a moving object such as a hand in an environment. With this understanding, we design several hand gestures which produce unique received signal variation patterns. Afterwards, we introduce the system implementation details about how different gestures are segmented and recognized from the LTE CSI signal stream and how they are mapped to the corresponding TV control commands. Finally, we present the demonstration process and the user interface.

\section{System Setup}

The gesture interaction system consists of one LTE (4G) base station and one $4 \mathrm{G}$ terminal, as shown in Figure 1. The LTE base station could be either an indoor femtocell or a macro cell, while the $4 \mathrm{G}$ terminal serves as a receiver that retrieves CSI from LTE downlink signals. In our prototype system, the

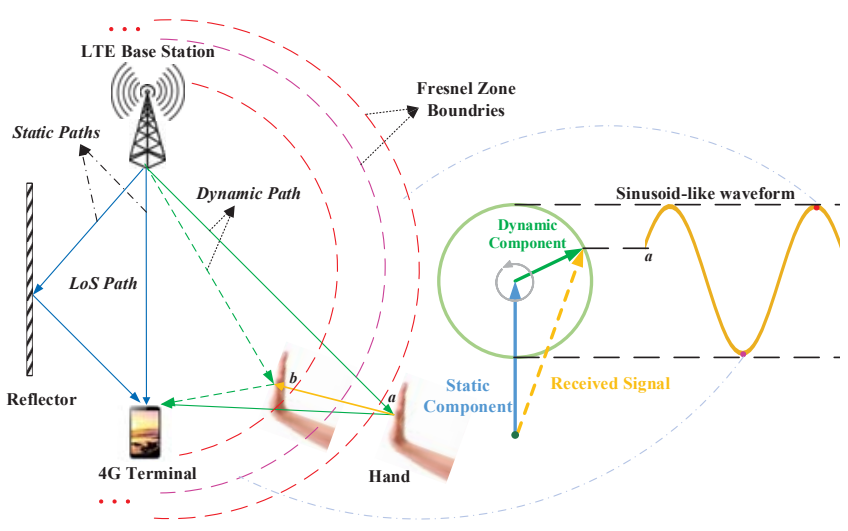

Figure 2: System setup and signal propagation model.

LTE base station is a commonly-used indoor femtocell (Figure 1a), while the receiver is a USRP B210 platform (Figure 1b) that can be configured to extract CSI from the received LTE frames. The receiver is equipped with two antennas and works on $2.165 \mathrm{GHz}$ band with $1.4 \mathrm{MHz}$ bandwidth. The $4 \mathrm{G}$ terminal receives CSI at the rate of 100 samples per second.

\section{LTE Signal Propagation Model}

As is shown in Figure 2, the LTE signal transmitted by LTE base station arrives at the $4 \mathrm{G}$ terminal through multiple paths, which can be grouped into two categories: static paths and dynamic path $[9,12]$. The static paths are composed of the Line-of-Sight (LoS) path and the reflected paths from the walls and the static objects in the environment, while the dynamic path is induced by the moving target such as a hand. The received signal is a superposition of signals from one dynamic path and multiple static paths. It can be expressed as an addition of a static phasor component and a dynamic phasor component on the complex plane. According to the Fresnel Zone model [9, 11-15], when the hand moves, the length of the dynamic signal path changes, and the dynamic phasor component rotates accordingly, resulting a sinusoid-like waveform. By measuring the phase change of the dynamic phasor component, we can estimate the change of the dynamic path quantitatively including both the length and direction. A hand gesture may involve a sequence of hand movements in different directions, producing an unique dynamic phasor change pattern, which results in an unique dynamic path change pattern. The patterns are robust to the environmental changes, which can be used as feature profiles to identify gestures.

\section{Gesture Design}

With the LTE signal propagation model, we know that different hand movements induce different dynamic path change 


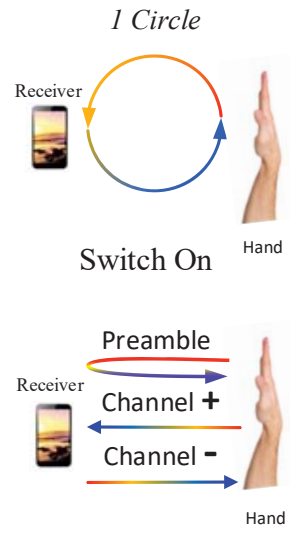

Channel Control

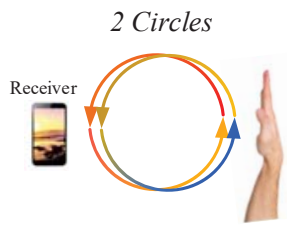

Switch Off Hand

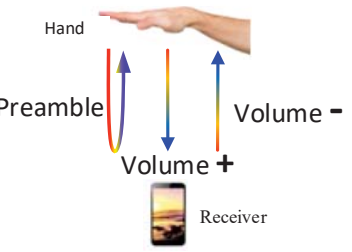

Volume Control
Figure 3: Designed hand gestures for remote TV control.

patterns in the received signals. Therefore, gesture recognition can be achieved without training as long as we design different gestures with unique and predictable signal patterns. Based on this principle, we design four hand gestures which produce different signal patterns for remote TV control. Each gesture is performed with the hand palm facing to the receiver and starts from the red points to the blue points in the directional arrow, as shown in Figure 3.

Specifically, the 'Switch On' gesture and the 'Switch Off' gesture for turning on and off TV are moving the hand on the side of the receiver in one circle and two circles, respectively. While that of controlling volume and channel are moving the hand towards and away from the receiver on the top and side of it, respectively. Note that preambles are designed as indicators for controlling the volume and channels, i.e., moving the hand straightly towards and away from the receiver on the top and side of it quickly. Once a preamble gesture is detected corresponding to the volume (or channel), users can perform the 'Volume Control' (or 'Channel Control') gesture subsequently to increase/decrease volume (or forward/backward channel).

When a user starts to perform hand gestures, the system first detects whether the generated signal variation pattern is corresponding to that of the 'Switch on' gesture. If they match well, the system issues the 'Switch On' command and starts the TV control process. Then it begins to recognize the next gesture by comparing the signal variation pattern with that of the gestures for 'Volume Control', 'Channel Control' and 'Switch Off'.

\section{System Design}

With the aforementioned system setup, the LTE signal propagation model and gesture design, the LTE-based gesture

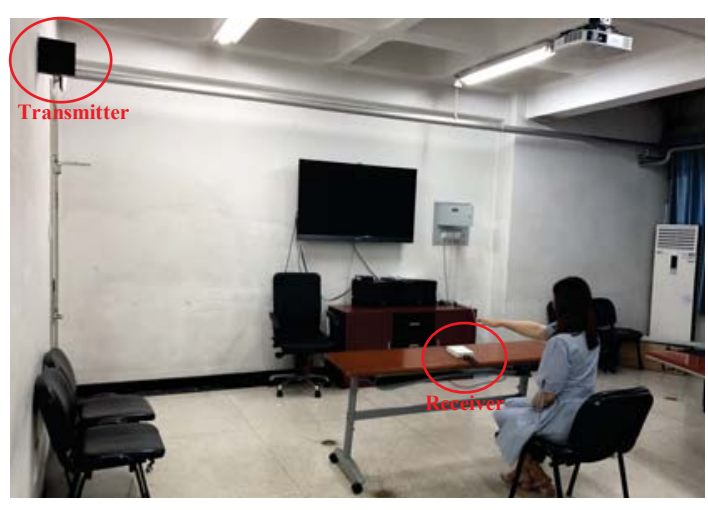

Figure 4: The demonstration environment.

interaction system consists of three functional modules: Motion Detection, Gesture Recognition and Control Command Generation.

(1) Motion detection. The first step is to segment gestures by detecting the start and the end samples within the LTE CSI readings. If there is no hand movement, the CSI amplitude variations are very small. But when there are hand movements, we observe much larger CSI amplitude variations. Therefore, the state transition of the CSI amplitude variation is utilized to identify the start and end of hand movement.

(2) Gesture Recognition. After detecting the hand movement, the corresponding dynamic path length change can be calculated, generating the corresponding hand movement length and direction pattern. As the generated signal variation pattern for each gesture is unique and predictable, we can thus employ the DTW (Dynamic Time Warping) algorithm to calculate the similarity between the segmented signal pattern and the reference signal patterns of the designed hand gestures. The reference signal pattern with the highest similarity is considered as the hand gesture performed.

(3) Control Command Generation. After recognizing the performed hand gestures, the system sends the corresponding control commands (i.e. switch on, switch off, etc.) to the TV remotely through a programmable infrared controller. After receiving the commands, the TV responses correspondingly.

\section{Demonstration Process}

With a $4 \mathrm{G}$ base station and a $4 \mathrm{G}$ terminal, we can build an LTE-based gesture interaction system for remote control of a TV set, as shown in Figure 4.

During the demonstration process, a user can do any daily activities freely, such as walking, exercising and sitting, etc.. When the user wants to watch TV, he/she can move close 


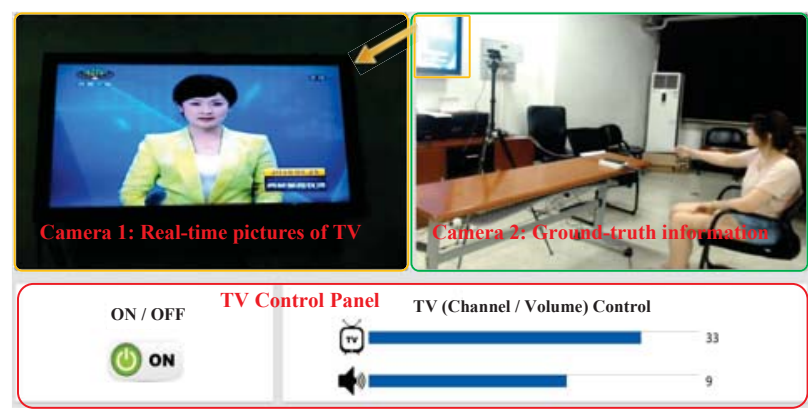

Figure 5: Graphical user interface of the LTE-based gesture interaction system.

to the 4G terminal and perform the 'Switch On' gesture to turn on the TV. After that, the user can either change the TV channel or adjust the volume with the corresponding hand gestures, which begin with a preamble gesture. Once the user wants to turn off the TV, he/she can perform the 'Switch Off' gesture. After the 'Switch Off' gesture is detected, the system sends 'Switch Off' command to TV and waits for the next 'Switch On' gesture. In addition to TV control, the system can also be applied to remotely control other appliances such as a refrigerator, a light. and an air-conditioner.

The user interface of the LTE-based gesture interaction system, as shown in Figure 5, is composed of three parts: (i) the TV status captured by a camera is displayed at the top-left of the interface. (ii) the ground truth information of the user's gesture captured by a camera is displayed at the top-right of the interface. (iii) the TV control commands corresponding to the recognized gestures are displayed at the bottom of the interface.

\section{CONCLUSION}

In this demo, we present the first LTE-based contactless gesture interaction system. It leverages the CSI extracted from the LTE downlink signal to recognize different hand gestures around a $4 \mathrm{G}$ terminal in a contactless way, which can be used for controlling home applicances remotely such as a TV set. We conduct extensive experiments to evaluate the effectiveness of the system with different users. The results show that the proposed system can recognize the hand gestures of different users reliably without training.

\section{ACKNOWLEDGMENTS}

We thank all the volunteers who participate in this work and the support of the NSFC Grant No.61572048, and Peking University Information Technology Institute (Tianjin Binhai).

\section{REFERENCES}

[1] Simone Di Domenico, Mauro De Sanctis, Ernestina Cianca, Paolo Colucci, and Giuseppe Bianchi. 2017. Lte-based passive device-free crowd density estimation. In 2017 IEEE International Conference on Communications (ICC). IEEE, 1-6.

[2] Marco Driusso, Chris Marshall, Mischa Sabathy, Fabian Knutti, Heinz Mathis, and Fulvio Babich. 2016. Indoor positioning using LTE signals. In 2016 International Conference on Indoor Positioning and Indoor Navigation (IPIN). IEEE, 1-8.

[3] Sidhant Gupta, Daniel Morris, Shwetak Patel, and Desney Tan. 2012. Soundwave: using the doppler effect to sense gestures. In Proceedings of the SIGCHI Conference on Human Factors in Computing Systems. ACM, 1911-1914.

[4] Xiang Li, Shengjie Li, Daqing Zhang, Jie Xiong, Yasha Wang, and Hong Mei. 2016. Dynamic-music: accurate device-free indoor localization. In Proceedings of the 2016 ACM International foint Conference on Pervasive and Ubiquitous Computing. ACM, 196-207.

[5] Xiang Li, Daqing Zhang, Qin Lv, Jie Xiong, Shengjie Li, Yue Zhang, and Hong Mei. 2017. IndoTrack: Device-free indoor human tracking with commodity Wi-Fi. Proceedings of the ACM on Interactive, Mobile, Wearable and Ubiquitous Technologies 1, 3 (2017), 72.

[6] Giulio Marin, Fabio Dominio, and Pietro Zanuttigh. 2014. Hand gesture recognition with leap motion and kinect devices. In 2014 IEEE International Conference on Image Processing (ICIP). IEEE, 1565-1569.

[7] Kai Niu, Fusang Zhang, Jie Xiong, Xiang Li, Enze Yi, and Daqing Zhang. 2018. Boosting fine-grained activity sensing by embracing wireless multipath effects. In Proceedings of the 14th International Conference on emerging Networking EXperiments and Technologies. ACM, 139-151.

[8] Giovanni Pecoraro, Simone Di Domenico, Ernestina Cianca, and Mauro De Sanctis. 2017. LTE signal fingerprinting localization based on CSI. In 2017 IEEE 13th International Conference on Wireless and Mobile Computing, Networking and Communications (WiMob). IEEE, 1-8.

[9] Hao Wang, Daqing Zhang, Junyi Ma, Yasha Wang, Yuxiang Wang, Dan Wu, Tao Gu, and Bing Xie. 2016. Human respiration detection with commodity wifi devices: do user location and body orientation matter?. In Proceedings of the 2016 ACM International foint Conference on Pervasive and Ubiquitous Computing. ACM, 25-36.

[10] Wei Wang, Alex X Liu, and Ke Sun. 2016. Device-free gesture tracking using acoustic signals. In Proceedings of the 22nd Annual International Conference on Mobile Computing and Networking. ACM, 82-94.

[11] Dan Wu, Daqing Zhang, Chenren Xu, Hao Wang, and Xiang Li. 2017. Device-free WiFi human sensing: From pattern-based to model-based approaches. IEEE Communications Magazine 55, 10 (2017), 91-97.

[12] Dan Wu, Daqing Zhang, Chenren Xu, Yasha Wang, and Hao Wang. 2016. WiDir: walking direction estimation using wireless signals. In Proceedings of the 2016 ACM international joint conference on pervasive and ubiquitous computing. ACM, 351-362.

[13] Daqing Zhang, Hao Wang, and Dan Wu. 2017. Toward centimeterscale human activity sensing with Wi-Fi signals. Computer 50, 1 (2017), 48-57.

[14] Fusang Zhang, Kai Niu, Jie Xiong, Beihong Jin, Tao Gu, Yuhang Jiang, and Daqing Zhang. 2019. Towards a Diffraction-based Sensing Approach on Human Activity Recognition. Proceedings of the ACM on Interactive, Mobile, Wearable and Ubiquitous Technologies 3, 1 (2019), 33.

[15] Fusang Zhang, Daqing Zhang, Jie Xiong, Hao Wang, Kai Niu, Beihong Jin, and Yuxiang Wang. 2018. From fresnel diffraction model to finegrained human respiration sensing with commodity wi-fi devices. Proceedings of the ACM on Interactive, Mobile, Wearable and Ubiquitous Technologies 2, 1 (2018), 53.

[16] Chen Zhao, Ke-Yu Chen, Md Tanvir Islam Aumi, Shwetak Patel, and Matthew S Reynolds. 2014. SideSwipe: detecting in-air gestures around mobile devices using actual GSM signal. In Proceedings of the 27th annual ACM symposium on User interface software and technology. ACM, 527-534. 